\title{
Méthode d'étude de la stabilité des ensembles convertisseurs-filtres
}

\author{
Philippe Barrade, Hubert Piquet et Yvon Chéron \\ Laboratoire d'Electrotechnique et d'Electronique Industrielle, ( ${ }^{*}$ ) I.N.P. Toulouse, \\ E.N.S.E.E.I.H.T., 2 Rue Charles Camichel, 31071 Toulouse Cedex, France
}

(Reçu le 10 juillet 1995, accepté le 17 octobre 1995)

\author{
PACS.02.60-x - Numerical approximation and analysis \\ PACS.02.70-c - Computational techniques \\ PACS.02.90+p - Other topics in mathematical methods in physics
}

\begin{abstract}
Résumé. - Tout système constitué d'un filtre chargé par un convertisseur statique qui absorbe une puissance constante est un système potentiellement instable. Nous développons dans cet article deux modèles pour l'étude de la stabilité, dont le point de départ est la représentation du convertisseur par une source de courant. Par une hypothèse petit signal, un premier modèle permet l'obtention de critères de stabilité par étude analytique puis numérique. Dans un deuxième temps, nous considérons un modèle fort signal, pour obtenir par calcul numérique le tracé de cycles limites de stabilité. Cela permet l'étude de l'aire de fonctionnement stable du système, en fonction des valeurs des paramètres du circuit. Enfin, à l'aide des fonctions de Ljapunov, nous définissons analytiquement des contours délimitant une partie des aires de fonctionnement stable déterminées par calcul numérique.
\end{abstract}

\begin{abstract}
Each system wich consists of a filter loaded by a converter absorbing a constant power is a system which is potentially unstable. In this document, models are developed for the study of the stability, in which current source is used to represent the converter. Using small signal hypothesis, the first model leads to analytical and numerical stability criteria, which are functions of the system parameters. In the second part, we use a large signal model, to obtain limit cycles of stability applying numerical calculation. This way we can study the influence of the system parameters on the area where the system is stable. Ljapunov theory is applied, to describe functions which represent part of the stable area we obtained by numerical calculation.
\end{abstract}

\section{Introduction}

L'utilisation de convertisseurs statiques munis de régulation et connectés sur un réseau d'alimentation continu nécessite l'utilisation de filtres. Ces filtres ont pour rôle d'atténuer, vis-à-vis du convertisseur, les variations de la tension réseau, et vis-à-vis du réseau les perturbations à haute fréquence en courant dues au découpage du convertisseur.

Si le convertisseur, régulé, alimente une charge fixe, la puissance qu'il fournit est alors constante. Cela implique que la puissance absorbée par le convertisseur est également constante [1]. Ceci est à l'origine de phénomènes d'instabilité au sein du filtre d'entrée. Un tel système peut

$\left(^{*}\right)$ U.R.A. au C.N.R.S. $N^{\circ} 847$

(C) Les Éditions de Physique 1996 

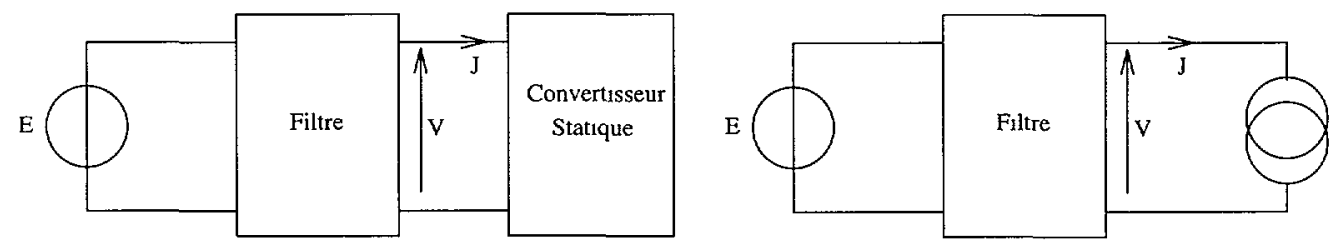

Fig. 1. - Modèle moyen.

[Averaged model.]

être instable, ou devenir instable face à une variation de la tension d'alimentation ou de la puissance absorbée par le convertisseur.

Nous proposons une modélisation permettant l'étude de la stabilité d'un tel système, dans une hypothèse petit puis fort signal.

\section{Définition du modèle}

2.1. DÉfinition GÉNÉRALE. - Nous considérons dans une première approche un ensemble filtre convertisseur, alimenté par une tension parfaitement continue.

Nous caractérisons dans un premier temps le filtre par sa fréquence de résonnance $f_{0}$. Celui-ci devant atténuer les perturbations haute fréquence en courant dues au convertisseur, la fréquence $f_{0}$ est nécessairement très inférieure à la fréquence de découpage $f_{\mathrm{c}}$. Nous pouvons ainsi, dans l'étude de la stabilité du filtre d'entrée, et donc dans un domaine de fréquence proche de $f_{0}$, représenter le convertisseur par une source de courant $J$ correspondant au courant absorbé (Fig. 1). Cela revient à raisonner sur un modèle moyen $[2,3]$, ce qui n'est pas une approximation étant donné l'écart séparant $f_{\mathrm{c}}$ et $f_{0}$. Le convertisseur absorbe une puissance $P_{0}$. Nous pouvons alors établir :

$$
J=\frac{P_{0}}{V}
$$

$P_{0}$ étant constante, nous pouvons écrire :

$$
J \delta V+V \delta J=0 \Rightarrow Z_{\mathrm{d}}=\frac{\delta V}{\delta J}=-\frac{V}{J}
$$

Où $Z_{\mathrm{d}}$ représente l'impédance d'entrée du convertisseur en régime dynamique. Cette impédance est négative et va donc provoquer les phénomènes d'instabilité.

Le circuit ainsi défini peut alors être mis en équation dans l'espace d'état :

$$
\left\{\begin{aligned}
\frac{\mathrm{d} x_{1}}{\mathrm{~d} t} & =a_{11} x_{1}+\cdot+a_{1 n} x_{n}+P_{1}\left(x_{1},\right. & \left., x_{n}\right)+A_{1} \\
\cdot & = & \\
\frac{\mathrm{d} x_{n}}{\mathrm{~d} t} & =a_{n 1} x_{1}+\cdot+a_{n n} x_{n}+P_{n}\left(x_{1},\right. & \left., x_{n}\right)+A_{n}
\end{aligned}\right.
$$

Où les variables $x_{\imath}$ sont les $n$ variables d'état du filtre d'entrée, qui sont les tensions capacitives et les courants inductifs. Le choix de ces données peut être réalisé à partir de considérations topologiques mises en ceuvre dans l'algorithme de Welsh [4]. Les $a_{\imath \jmath}$ sont des fonctions des paramètres du système (inductances, capacités, et éventuellement résistances du filtre).

Les termes $P_{\imath}\left(x_{1}, \quad, x_{n}\right)$ représentent les termes non-linéaires du système considéré.

Quant aux $A_{\imath}$, il s'agit de termes constants qui représentent les tensions ou courants imposés au système. 


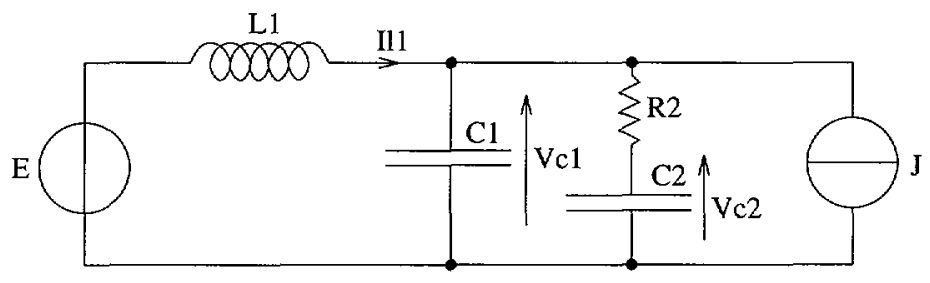

Fig. 2. - Filtre avec cellule de stabilisation.

[Filter with stabilizing circuit.]

2.2. EXEMPLE. - Nous considérons un filtre élémentaire (Fig. 2) constitué d'une cellule $L_{1} C_{1}$. La prise en compte des pertes propres dans ces composants ne permettant d'assurer la stabilité que pour un nombre de points de fonctionnement trop limité (convertisseur absorbant une puissance proche de zéro), $L_{1}$ et $C_{1}$ seront donc considérés comme parfaits, c'est-à-dire sans résistance parallèle ou série. Comme exposé précédemment, le convertisseur statique est représenté par une source de courant $J$. Sous l'hypothèse que le convertisseur absorbe une puissance constante $P_{0}$, nous avons démontré (Éq. 2) que l'impédance d'entrée $Z_{\mathrm{d}}$ du convertisseur est de signe négatif. L'étude de la fonction de transfert du filtre $L_{1} C_{1}$ chargé par une telle impédance montre que l'amortissement du système serait négatif. Il y aurait par conséquence instabilité. Une solution consiste à ajouter un élément dissipatif (résistance $R_{2}$ ) en parallèle sur $C_{1}$, afin que le filtre soit chargé par l'impédance équivalente représentée par $R_{2}$ en parallèle avec $Z_{\mathrm{d}}$, de sorte que cette impédance soit positive. La stabilité pouvant être assurée, il convient alors de contrôler la puissance dissipée par $R_{2}$, afin qu'il n'y ait de pertes que si le filtre $L_{1} C_{1}$ oscille. Pour cette raison, on insère en série avec $R_{2}$ une capacité $C_{2}[5]$.

Le système ainsi défini, la mise en équation conduit au système d'équations suivant:

$$
\left\{\begin{aligned}
\frac{\mathrm{d} V_{\mathrm{c}_{1}}}{\mathrm{~d} t} & =-\frac{V_{\mathrm{c}_{1}}}{C_{1} R_{2}}+\frac{V_{\mathrm{c}_{2}}}{C_{1} R_{2}}+\frac{I_{1}}{C_{1}}-\frac{P_{0}}{C_{1} V_{\mathrm{c}_{1}}} \\
\frac{\mathrm{d} V_{\mathrm{c}_{2}}}{\mathrm{~d} t} & =\frac{V_{\mathrm{c}_{1}}}{C_{2} R_{2}}-\frac{V_{\mathrm{c}_{2}}}{C_{2} R_{2}} \\
\frac{\mathrm{d} I_{1}}{\mathrm{~d} t} & =-\frac{V_{\mathrm{c}_{1}}}{L_{1}}+\frac{E}{L_{1}}
\end{aligned}\right.
$$

Dans cet exemple, et conformément aux notations définies dans l'équation (3), on obtient :

$$
\begin{array}{ccc}
P_{1}\left(V_{\mathrm{c}_{1}}, V_{\mathrm{c}_{2}}, I_{1}\right)=-\frac{P_{0}}{C_{1} V_{\mathrm{c}_{1}}} & \text { et } & P_{2}\left(V_{\mathrm{c}_{1}}, V_{\mathrm{c}_{2}}, I_{1}\right)=P_{3}\left(V_{\mathrm{c}_{1}}, V_{\mathrm{c}_{2}}, I_{1}\right)=0 \\
A_{1}=A_{2}=0 & \text { et } & A_{3}=\frac{E}{L_{1}}
\end{array}
$$

La validité de ce modèle est mise en évidence sur le tracé suivant (Fig. 3), où nous comparons la résolution du système d'équations (4) effectuée à l'aide du logiciel MATLAB avec le résultat issu d'un outil de simulation de type circuit (SUCCESS). Le convertisseur considéré est ici un hacheur dévolteur, fonctionnant à la fréquence de découpage de $10 \mathrm{kHz}$, et absorbant une puissance constante de $4 \mathrm{~kW}$. Les éléments du filtre ont pour valeur: $L_{1}=10 \mathrm{mH}, C_{1}=10 \mu \mathrm{F}$, $R_{2}=40 \Omega$ et $C_{2}=50 \mu \mathrm{F}$. Enfin, la source de tension $E$, considérée comme unidirectionnelle en courant, a pour valeur $500 \mathrm{~V}$. 

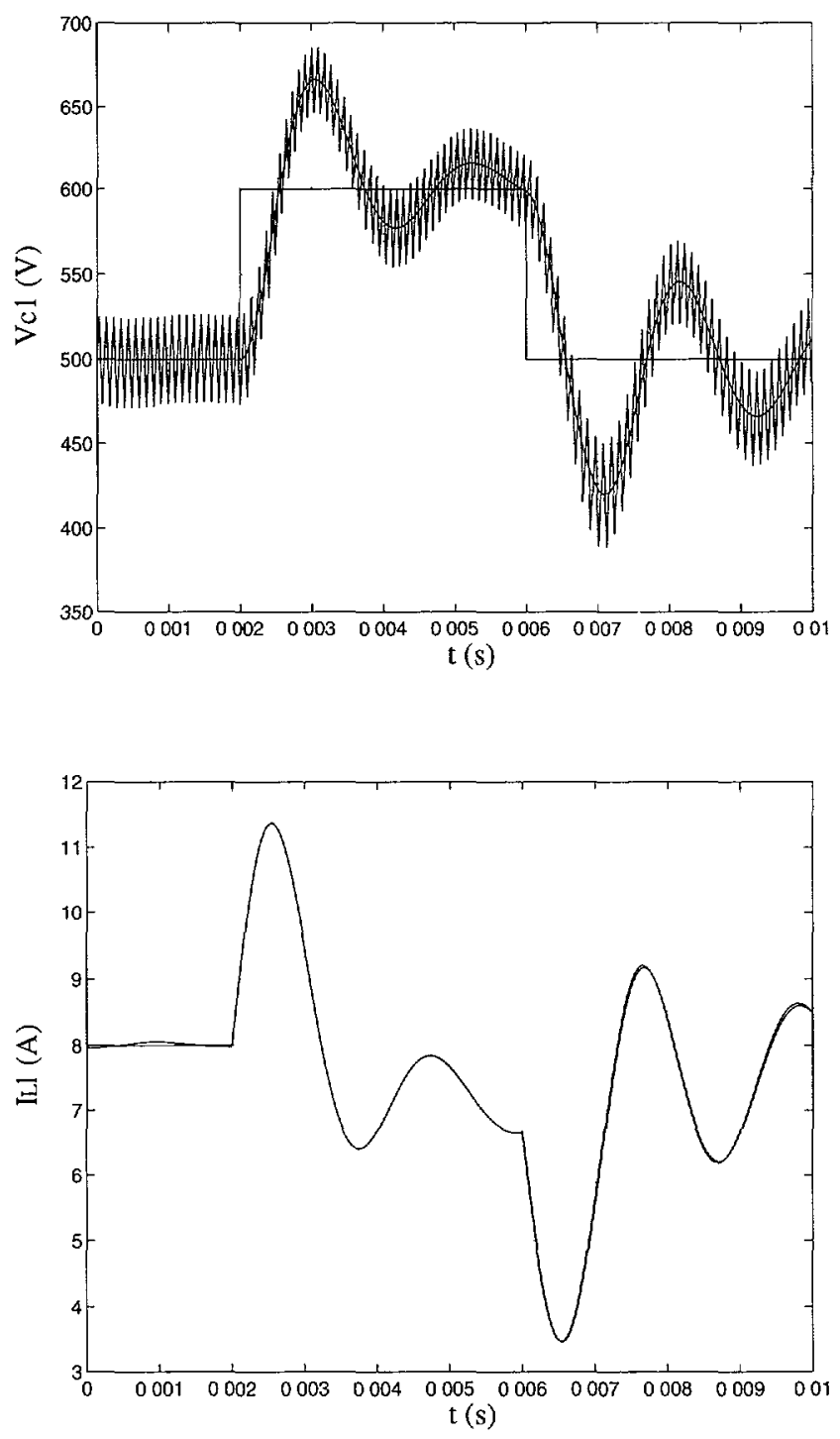

Fig. 3. - Validité du modèle proposé.

[Validity of the proposed model.]

Pour une variation de la tension d'alimentation $E(500 \mathrm{~V}-600 \mathrm{~V}-500 \mathrm{~V})$, on compare la tension en sortie du filtre à la valeur obtenue à partir du modèle proposé. Ce dernier ne rend pas compte des variations de la tension de sortie du filtre dues au découpage du convertisseur, mais suit parfaitement, à l'échelle de la fréquence de résonnance du filtre, les variations de cette tension. Pour le courant dans l'inductance $L_{1}$, les ondulations liées au découpage du hacheur sont négligeables, et les deux courbes sont confondues. La représentation du convertisseur par une source de courant absorbant une puissance constante à l'échelle de la fréquence de résonnance du filtre est ici pleinement justifiée. 


\section{Modèle petit signal}

3.1. CAS GÉNÉRAL. - Face à la non-linéarité du système considéré (termes $P_{\imath}\left(x_{1}, \quad, x_{n}\right)$ ), une première approche des problèmes de stabilité peut être effectuée en étudiant le comportement du système au voisinage d'un point de fonctionnement [2,3]. Ceci peut être réalisé en linéarisant le système d'équations d'état autour du point de régime forcé imposé au filtre par l'alimentation $E$ d'une part, et par le courant $J$ (donc par la puissance $P_{0}$ ) d'autre part. Pour ce faire, on effectue, pour tous les termes $P_{2}\left(x_{1}, \quad, x_{n}\right)$, un développement limité au premier ordre. Le système d'équations d'état peut alors s'écrire sous la forme :

$$
\left\{\begin{aligned}
\frac{\mathrm{d} x_{1}}{\mathrm{~d} t} & =b_{11} x_{1}+\cdot+b_{1 n} x_{n}+B_{1} \\
\frac{\mathrm{d} x_{n}}{\mathrm{~d} t} & =b_{n 1} x_{1}+\cdot+b_{n n} x_{n}+B_{n}
\end{aligned}\right.
$$

Où les termes $B_{\imath}$ représentent les termes constants. Ce système d'équations linéaires permet le calcul de la fonction de transfert $F(p)$. L'étude des pôles de $F(p)$ va permettre de se prononcer sur la stabilité du système linéarisé. Deux cas de figure peuvent se présenter :

- Ou bien le système est d'ordre 2 : il est aisé dans ce cas de calculer les pôles en fonction des paramètres du système (valeurs de $E, P_{0}$, des inductances et capacités du filtre). Pour assurer la stabilité, on doit s'assurer que les deux pôles de $F(p)$ sont à partie réelle négative.

- Ou bien le système est d'ordre supérieur à 2 : sauf cas particulier, il est difficile d'obtenir dans ce cas une équation analytique donnant le signe des parties réelles des pôles. Seule une étude numérique est alors possible, où l'on étudie, en fonction des valeurs des paramètres du système, l'évolution du signe des parties réelles des pôles.

Nous disposons cependant pour le second cas de figure des critères de Hurwitz [6] donnant des conditions nécessaires et suffisantes quant au signe des parties réelles des pôles.

Soit $D(p)$ le dénominateur de la fonction de transfert $F(p)$, de la forme :

$$
D(p)=a_{0} p^{n}+a_{1} p^{n-1}+\cdot+a_{n-1} p+a_{n}
$$

On suppose $a_{0}$ positif, sinon on divise $D(p)$ par -1 . On forme alors le tableau suivant (Tab. I) :

Tableau I. - Détermination des critères de Hurwitz

[Determination of Hurwitz criteria.]

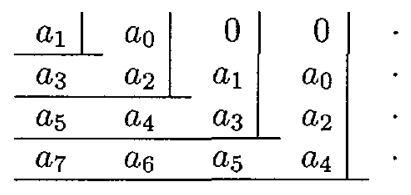

On calcule alors les déterminants repérés par les traits continus. Le critère de Hurwitz établit une condition nécessaire et suffisante de stabilité, qui est que tous ces déterminants soient positifs. La stabilité est ici considérée au sens automatique du terme, c'est-à-dire que tous les 
pôles de $F(p)$ sont à partie réelle négative.

$$
\begin{aligned}
a_{1} & >0 \\
a_{1} a_{2}-a_{3} a_{0} & >0 \\
a_{1}\left(a_{2} a_{3}-a_{4} a_{1}\right)-a_{0}\left(a_{3} a_{3}-a_{5} a_{1}\right) & >0 \\
& >0
\end{aligned}
$$

Nous disposons donc, par l'application de tels critères, d'un jeu d'expression reliant les paramètres du système étudié, qui permet l'étude de l'influence de ces paramètres sur le comportement du système.

3.2. Application. - Nous considérons le même système qu'au paragraphe 2.2. Le régime forcé imposé au filtre en régime permanent est $E$ pour la tension aux bornes des capacités $C_{1}$ et $C_{2}$, et $\frac{P_{0}}{E}$ pour le courant dans l'inductance $L_{1}$. Les valeurs de $L_{1}$ et de $C_{1}$ sont déjà définies par des critères liés à l'ondulation de courant dans $L_{1}$ et à l'ondulation de tension aux bornes de $C_{1}$. Le problème que l'on se pose est de déterminer les paramètres du circuit additionnel $R_{2} C_{2}$ pour assurer la stabilité. $A$ cet effet, nous mettons en œuvre dans cet exemple les critères exposés plus haut.

La non-linéarité du système étant due au terme $P_{1}\left(V_{\mathrm{c}_{1}}, V_{\mathrm{c}_{2}}, I_{1}\right)$ (Éq. (5)), on en calcule le développement limité pour $V_{\mathrm{c}_{1}}$ proche de $E$, en ne considérant que les termes de degrés inférieurs ou égaux à 2 .

$$
P_{2}\left(V_{\mathrm{c}_{1}}, V_{\mathrm{c}_{2}}, I_{1}\right) \simeq-\frac{P_{0}}{C_{1}}\left(\frac{1}{E}-\frac{V_{\mathrm{c}_{1}}-E}{E^{2}}\right)
$$

On reporte ce terme dans les équations (4) et l'on obtient les équations suivantes, relatives au système linéarisé :

$$
\left(\begin{array}{c}
\dot{V}_{c_{1}} \\
\dot{V}_{c_{2}} \\
\dot{I}_{1}
\end{array}\right)=\left(\begin{array}{ccc}
\frac{-1}{R_{2} C_{1}}+\frac{P_{0}}{R_{2} C_{1}} & \frac{1}{R_{2} C_{1}} & \frac{1}{C_{1}} \\
\frac{1}{R_{2} C_{2}} & -\frac{1}{R_{2} C_{2}} & 0 \\
\frac{1}{L_{1}} & 0 & 0
\end{array}\right)\left(\begin{array}{c}
V_{c_{1}} \\
V_{\mathrm{c}_{2}} \\
I_{1}
\end{array}\right)+\left(\begin{array}{c}
\frac{-2 P_{0}}{C_{1} E^{2}} \\
0 \\
\frac{1}{L_{1}}
\end{array}\right) E
$$

On est alors en mesure de déterminer la fonction de transfert du système, que nous définirons ici par :

$$
F(p)=\frac{V_{\mathrm{c}_{1}}(p)}{E(p)}
$$

Nous nous contenterons ici de donner comme résultat l'expression du dénominateur de $F(p)$ :

$$
\begin{aligned}
D(p)= & p^{3}+p^{2}\left(\frac{1}{R_{2} C_{1}}+\frac{1}{R_{2} C_{2}}-\frac{P_{0}}{C_{1} E^{2}}\right) \\
& +p\left(\frac{1}{L_{1} C_{1}}-\frac{P_{0}}{R_{2} C_{1} C_{2} E^{2}}\right)+\frac{1}{R_{2} L_{1} C_{1} C_{2}}
\end{aligned}
$$

L'application du critère de Hurwitz à ce polynôme donne les deux critères suivants :

$$
\begin{gathered}
\frac{1}{C_{1} R_{2}}+\frac{1}{C_{2} R_{2}}-\frac{P_{0}}{C_{1} E^{2}}>0 \\
\left(\frac{1}{C_{1} R_{2}}+\frac{1}{C_{2} R_{2}}-\frac{P_{0}}{C_{1} E^{2}}\right)\left(\frac{1}{C_{1} L_{1}}-\frac{P_{0}}{C_{1} C_{2} E^{2} R_{2}}\right)-\frac{1}{C_{1} C_{2} L_{1} R_{2}}>0
\end{gathered}
$$




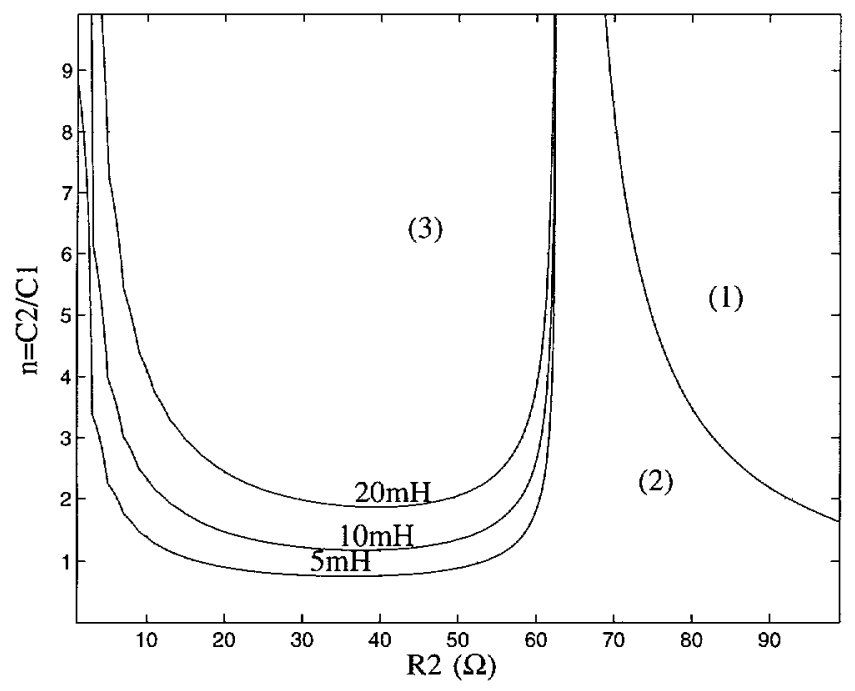

Fig. 4. - Application du critère de Hurwitz : dimensionnement des éléments de stabilisation.

[Application of Hurwitz criterion: determination of the stabilizing components.]

Le respect de ces deux équations nous assure du signe des parties réelles des racines de $D(p)$, qui doit être négatif. De tels critères peuvent cependant être difficiles à analyser, de par la complexité de leur expression. Il est alors toujours possible de procéder au tracé d'abaques permettant, pour $L_{1}, C_{1}, \mathrm{E}$ et $P_{0}$ fixés, de déterminer les valeurs possibles de $R_{2}$ et $C_{2}$ qui vérifient les relations (13) et (14). On pose :

$$
C_{2}=n C_{1}
$$

Nous considérons les valeurs suivantes : $C_{1}=10 \mu \mathrm{F}, E=500 \mathrm{~V}$ et $P_{0}=4000 \mathrm{~W}$.

Pour ces valeurs, nous traçons dans le plan $\left(R_{2}, n\right)$ les lieux des points délimitant l'ensemble des points pour lesquels les deux critères sont vérifiés (Fig. 4) pour trois valeurs de $L_{1}(5 \mathrm{mH}$, $10 \mathrm{mH}, 20 \mathrm{mH}$ ).

Les tracés correspondent aux lieux des points où les expressions (13) et (14) sont nulles. Trois zones du plan $\left(R_{2}, n\right)$ sont ainsi délimitées :

- zone (1) : aucun des critères (13) et (14) ne sont vérifiés.

- zone (2) : seul le critère (13) est vérifié.

- zone (3) : les deux critères sont vérifiés.

Pour tous les points compris dans la zone où les deux critères sont vérifiés, on est en mesure de garantir la stabilité du système linéarisé. L'influence de la valeur de $L_{1}$ sur le critère (14) est également mise en évidence. En effet, plus $L_{1}$ est faible, plus le domaine des valeurs de $R_{2}$ et $C_{2}$ assurant la stabilité est étendu.

Ce modèle permet ainsi l'obtention de critères de stabilité mettant en relation les divers paramètres du système $\left(L_{1}, C_{1}, C_{2}, R_{2}\right)$, ainsi que des données issues du cahier des charges $\left(E, P_{0}\right)$.

Cependant, si le respect de tels critères permet d'assurer que les parties réelles des pôles sont négatives, nous $\mathrm{n}$ avons aucun renseignement ni sur leurs valeurs, ni sur celles des parties 


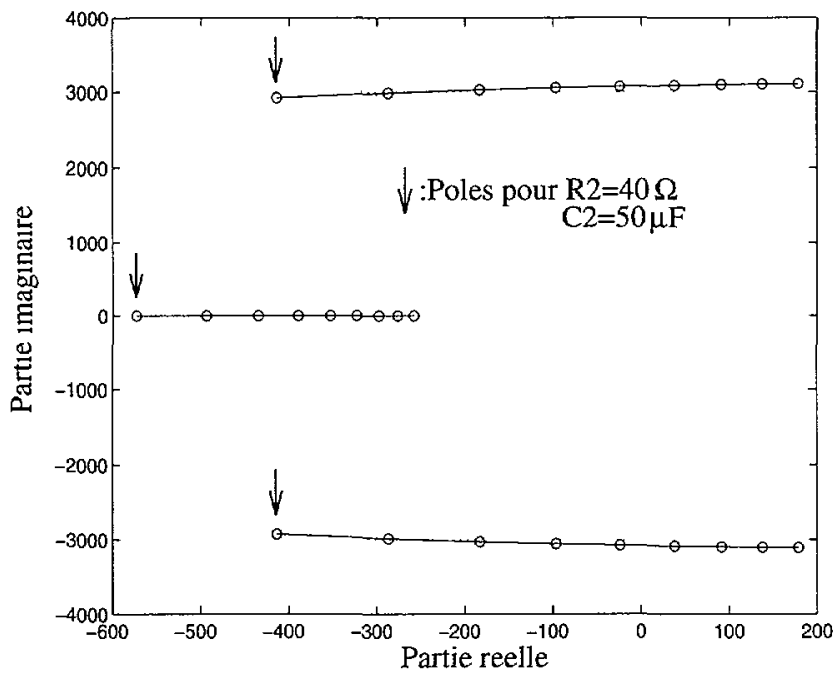

Fig. 5. - Valeurs des pôles du système en fonction de $R_{2}$.

[Poles values as a function of $R_{2}$.]

imaginaires si elles existent. Il convient alors, pour les couples $R_{2} C_{2}$ retenus, de préciser les valeurs des pôles, afin d'en déduire l'amortissement du système.

A titre d'exemple, nous traçons dans le plan de Laplace les pôles du système pour $L_{1}=10 \mathrm{mH}, C_{1}=10 \mu \mathrm{F}, E=500 \mathrm{~V}, P_{0}=4000 \mathrm{~W}, C_{2}=50 \mu \mathrm{F}$ et $R_{2}$ variant de 40 à $80 \Omega$ (Fig. 5).

Pour $R_{2}$ compris entre 62 et $80 \Omega$, les pôles imaginaires ont une partie réelle positive, ce qui confirme les renseignements obtenus à l'aide des critères de Hurwitz. Pour $R_{2}=40 \Omega$, les pôles dominant sont les deux pôles complexes conjugués, à partir desquels on peut déduire, par exemple, l'amortissement du système. Soient $p_{1}$ et $p_{2}$ ces pôles :

$$
R_{\mathrm{e}}\left(p_{1}, p_{2}\right)=-z \omega_{n}
$$

Où $z$ représente la facteur d'amortissement du système et $\omega_{n}$ la fréquence de résonnance du filtre.

Pour $R_{2}=40 \Omega, R_{\mathrm{e}}\left(p_{1}, p_{2}\right)=-413,5$ et $\omega_{n}=3160 \mathrm{rad} \mathrm{s}^{-1}$ on obtient un facteur d'amortissement $z=0,13$.

Une fois la stabilité ainsi assurée, il convient cependant, pour $R_{2}$ et $C_{2}$ répondant aux critères (13) et (14), de s'assurer du respect du cahier des charges imposant, par exemple, l'ondulation de courant dans $L_{1}$ et l'ondulation de tension aux bornes de $C_{1}$.

\section{Modèle fort signal}

4.1. Mise EN Évidence DU PROBLÈme. - Le modèle petit signal précédemment établi ne nous renseigne sur la stabilité du système que dans le voisinage du point de fonctionnement considéré. Les critères établis, s'ils permettent de garantir la stabilité dans cette hypothèse, ne permettent pas de se prononcer sur le comportement du filtre face à des perturbations réseau ou des échelons de charge de forte amplitude. 
En effet, si l'on résout, à partir de procédures de calcul numérique, le système d'équations (4), pour des conditions initiales différentes de la position d'équilibre, on obtient le résultat suivant (Fig. 6) :

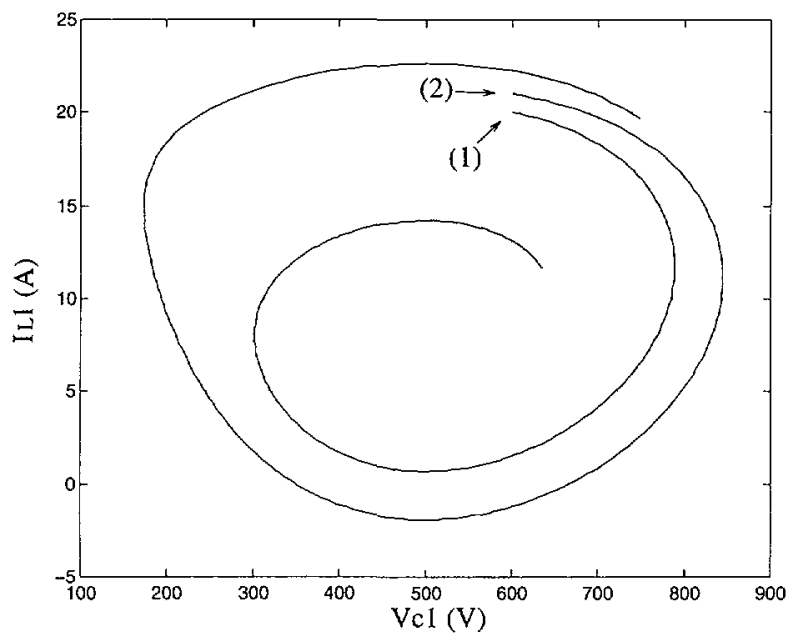

Fig. 6. - Comportement du système suivant les conditions initiales appliquées.

[System behavior as a function of the applied initial conditions.]

Les valeurs de $L_{1}, C_{1}, R_{2}, C_{2}$ ont été choisies de façon à satisfaire les critères établis précédemment dans l'hypothèse petit signal : $L_{1}=10 \mathrm{mH}, C_{1}=10 \mu \mathrm{F}, C_{2}=50 \mu \mathrm{F}, R_{2}=40 \Omega$. Pour $E=500 \mathrm{~V}$ et $P_{0}=4000 \mathrm{~W}$, le point d'équilibre est défini par $V_{\mathrm{c}_{1}}=V_{\mathrm{c}_{2}}=500 \mathrm{~V}$ et $I_{l_{1}}=8 \mathrm{~A}$.

- pour le tracé (1), correspondant aux conditions initiales $V_{\mathrm{c}_{1}}=600 \mathrm{~V}, V_{\mathrm{c}_{2}}=550 \mathrm{~V}$ et $I_{1_{1}}=20 \mathrm{~A}$, le système converge, c'est-à-dire qu'à l'issue d'une période d'oscillation, la distance entre le point de fonctionnement et le point d'équilibre a décru. Le système est bien stable.

- pour le tracé (2), correspondant aux conditions initiales $V_{\mathrm{c}_{1}}=600 \mathrm{~V}, V_{\mathrm{c}_{2}}=552 \mathrm{~V}$ et $I_{I_{1}}=21 \mathrm{~A}$, les conditions initiales sont plus éloignées du point d'équilibre que dans le cas (1), et l'on constate que le système diverge.

On met ainsi en évidence l'existence d'une zone de fonctionnement stable représentant le domaine des variations possibles des variables d'état sans que le système devienne instable, et d'une zone de fonctionnement instable, pour laquelle le système diverge. Notre objectif est de définir la frontière délimitant ces deux zones, appelée cycle limite de stabilité. La détermination de ce contour fermé, qui comprend le point de fonctionnement souhaité, permet de définir la stabilité du circuit de façon globale, et non plus seulement dans le voisinage du point de fonctionnement.

4.2. RECherChE DES CYCLES LIMITES - - Le problème qui se pose est donc de définir la zone du plan de phase dans laquelle le système reste stable et celle dans laquelle le système diverge. Nous ne pouvons, dans ce cadre, utiliser les méthodes classiques, puisque le système 


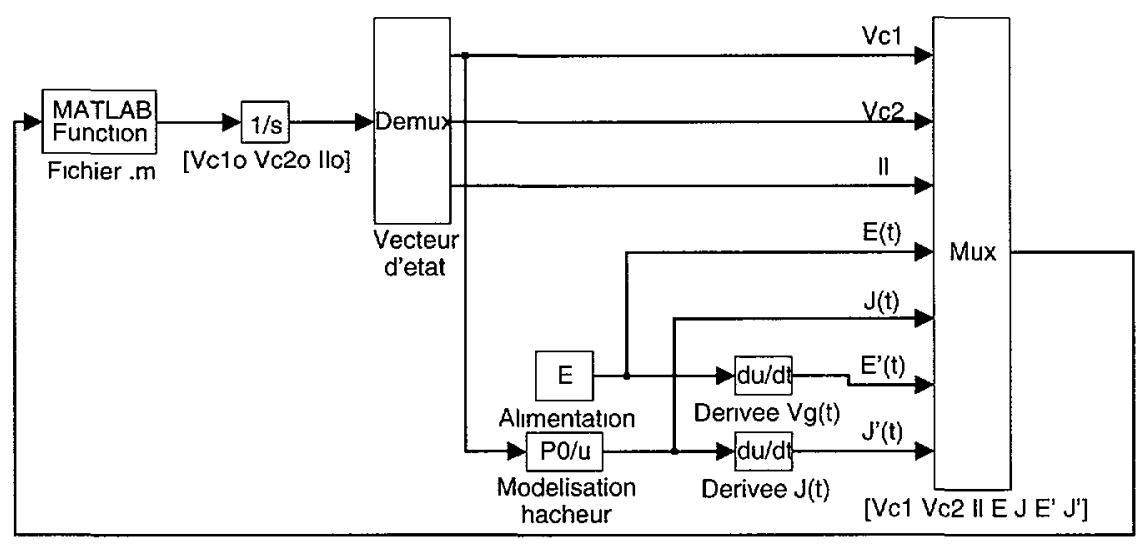

Fig. 7. - Résolution numérique.

[Numerical calculation.]

est non linéaire. De même, ce résultat ne peut être fourni par le modèle petit signal puisque celui-ci n'est valable que pour de faibles variations autour du point de fonctionnement, ainsi que nous l'avons mis en évidence précédemment. Le tracé du cycle limite de stabilité ne peut être obtenu que par l'étude du modèle fort signal (Éqs. 3). Pour ce faire, on intègre numériquement ce système d'équations.

La caractéristique principale du contour recherché est qu'il s'agit d'un cycle limite ınstable, dans le sens où toute trajectoire décrite par le point de fonctionnement s'éloigne de ce cycle. Si la trajectoire est interne au cycle, on tend vers le point d'équilibre; si la trajectoire est externe au cycle, le système diverge. Si l'on considère le système adjoint au système étudié (on affecte toutes les dérivées des variables d'état du filtre d'un signe moins), alors le cycle limite garde le même contour, mais devient cycle limite stable : toute trajectoire tend vers ce cycle. Ainsi, quelles que soient les conditions initiales choisies, le système convergera vers son cycle limite $[6,7]$.

Nous avons mis au point pour cette recherche un ensemble d'outils informatiques, permettant, à partir de la définition de la topologie d'un circuit et des valeurs des composants mis en cuvre, de générer automatiquement le système d'équations (3). Le fichier obtenu est alors intégré dans un système de calcul numérique (bloc "MATLAB Function", Fig. 7), défini sous le logiciel MATLAB, qui permet de réaliser la procédure de recherche de cycle limite que nous venons d'exposer.

A titre d'illustration (Fig. 8), nous présentons le tracé d'un cycle limite correspondant au système défini au paragraphe 2.2 , pour $L_{1}, C_{1}, R_{2}, C_{2}, E, P_{0}$ correspondant aux valeurs fixées pour la simulation précédente (contour en traits pleins, Fig. 6). La courbe en pointillés correspond à la trajectoire suivie par le couple de points $\left(V_{c_{1}}, I_{l_{1}}\right)$ à partir de conditions initiales différentes de l'équilibre, lorsque l'on injecte dans le bloc "MATLAB Function" (Fig. 7) le système adjoint au système considéré (Éqs. 3). Le sens de parcours se fait dans le sens trigonométrique, et la trajectoire tend vers le cycle limite.

Il convient de remarquer que s'il est possible de construire le cycle limite pour toute topologie, le résultat dépend du choix préalable des valeurs des composants. Le résultat obtenu ne permet done pas le dimensionnement du filtre a priori. Pour cette raison, on doit alors rechercher l'expression analytique de ce cycle, afin d'étudier l'influence des divers paramètres sur celui-ci. 


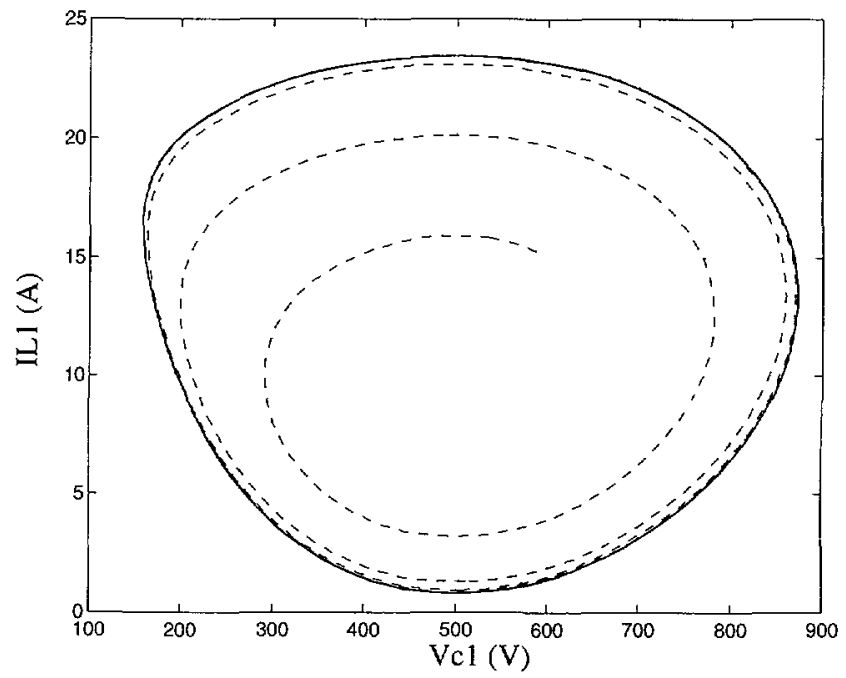

Fig. 8. - Détermination de cycle limite.

[Determination of the limit cycle.]

De plus, il convient de préciser, en considérant le cycle limite tracé (Fig. 8), que si on peut garantir la stabilité du système tant que le point de fonctionnement reste compris à l'intérieur du cycle limite, les excursions passibles de $V_{c_{1}}$ et $l_{l_{1}}$ autour du point d'équilibre peuvent être importantes. Outre le fait d'assurer la stabilité, il faudra par la suite être en mesure de limiter les variations de ces grandeurs à l'intérieur même de ce cycle limite.

4.3. EXPRESSION ANALYTIQUE DU CYCLE LIMITE. - L'objectif que nous venons de fixer nous conduit à l'utilisation des fonctions de Ljapunov [7].

Considérant le système d'équations (3), on définit une fonction $V$, dite de Ljapunov, qui doit être fonction des variables d'état du système $\left(V\left(x_{1}, x_{2}, \cdot, x_{n}\right)\right)$. Cette fonction doit être de valeur nulle lorsque les variables d'état ont pour valeurs celles de la position d'équilibre $\left(\left(V\left(X_{1}, X_{2},{ }^{\cdot}, X_{n}\right)=0\right)\right.$. De plus, les contours $V\left(x_{1}, x_{2}, \cdot, x_{n}\right)=K$ doivent être des contours fermés, tels que si $K_{1}<K_{2}$, alors la surface $V=K_{1}$ soit entièrement située à l'intérieur de la surface $V=K_{2}$.

Dans le cadre de notre étude, on définit généralement $V\left(x_{1}, x_{2}, \cdot, x_{n}\right)$ telle qu'elle soit une fonction de type quadratique :

$$
V\left(x_{1}, x_{2}, \cdot, x_{n}\right)=K_{1}\left(x_{1}-X_{1}\right)^{2}+K_{2}\left(x_{2}-X_{2}\right)^{2}+\cdot+K_{n}\left(x_{n}-X_{n}\right)^{2}
$$

Les variables d'état représentant les courants dans les inductances et les tensions aux bornes des capacités, cela revient à considérer l'énergie mise en jeu par les variations de $\left(x_{1}, x_{2},{ }^{\cdot}, x_{n}\right)$ autour de $\left(X_{1}, X_{2},{ }^{\prime}, X_{n}\right)$. On calcule alors la dérivée de $V$ par rapport an temps :

$$
\frac{\mathrm{d} V}{\mathrm{~d} t}=2 K_{1}\left(x_{1}-X_{1}\right) \frac{\mathrm{d} x_{1}}{\mathrm{~d} t}+2 K_{2}\left(x_{2}-X_{2}\right) \frac{\mathrm{d} x_{2}}{\mathrm{~d} t}+\cdot+2 K_{n}\left(x_{n}-X_{n}\right) \frac{\mathrm{d} x_{n}}{\mathrm{~d} t}
$$

On reporte dans cette relation les expressions des dérivées définies dans le système (3). 
A l'équilibre, $\left(x_{1}, x_{2}, \cdot, x_{n}\right)=\left(X_{1}, X_{2}, \cdot, X_{n}\right), V$ et sa dérivée sont nulles. Suite à une perturbation qui éloigne le point de fonctionnement de l'équilibre, $\left(x_{1}, x_{2}, \cdot, x_{n}\right) \neq$ $\left(X_{1}, X_{2}, \cdots, X_{n}\right), V$ devient positive. Si la dérivée de $V$ est de valeur négative, $V$ décroît jusqu'à redevenir nulle au point d'équilibre. A l'opposé, si la dérivée de $V$ est de signe positif, cela entraîne une augmentation de $V$, c'est-à-dire une augmentation de l'écart séparant $\left(x_{1}, x_{2} \cdot \cdot, x_{n}\right)$ de $\left(X_{1}, X_{2}, \cdot, X_{n}\right)$.

Pour déterminer l'aire de stabilité du système, il s'agit donc de déterminer le contour $V=K$ le plus grand pour lequel $V$ et sa dérivée par rapport au temps sont de signes opposés : cette condition suffisante de stabilité permet, à l'intérieur de ce contour, de garantir la stabilité du système.

4.4. Exemple D'Application. - Nous considérons la topologie suivante (Fig. 9):

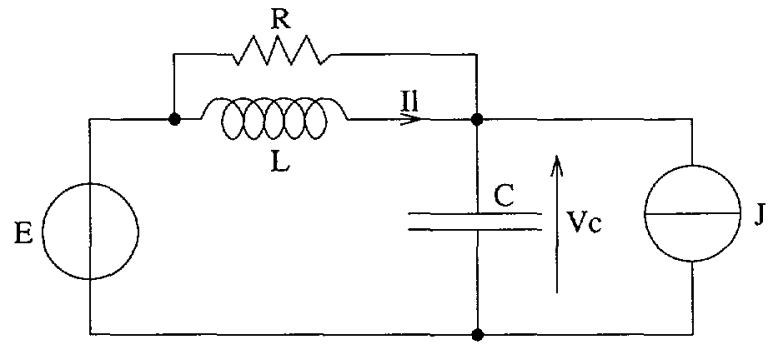

Fig. 9. - Filtre avec résistance d'amortissement.

[Filter with damping resistance.]

Le système d'équations d'état relatif à ce système est :

$$
\left\{\begin{aligned}
\frac{\mathrm{d} V_{c}}{\mathrm{~d} t} & =-\frac{V_{\mathrm{c}}}{C R}+\frac{I_{1}}{C}-\frac{P_{0}}{C V_{\mathrm{c}}}+\frac{E}{C R} \\
\frac{\mathrm{d} I_{1}}{\mathrm{~d} t} & =-\frac{V_{\mathrm{c}}}{L}+\frac{E}{L}
\end{aligned}\right.
$$

Comme exposé dans le paragraphe 4.1, nous avons dans un premier temps procédé au tracé du cycle limite de stabilité pour $R=40 \Omega, L=10 \mathrm{mH}, C=10 \mu \mathrm{F}, E=500 \mathrm{~V}$ et $P_{0}=4000 \mathrm{~W}$ (contour(1) Fig. 10).

Afin de définir l'expression analytique du cycle limite mis en évidence, nous posons comme définition de la fonction $V$ :

$$
V=\frac{1}{2} C\left(V_{\mathrm{c}}-E\right)^{2}+\frac{1}{2} L\left(I_{1}-\frac{P_{0}}{E}\right)^{2}
$$

Le calcul de la dérivée de $V$ donne :

$$
\frac{\mathrm{d} V}{\mathrm{~d} t}=C\left(V_{\mathrm{c}}-E\right) \frac{\mathrm{d} V_{\mathrm{c}}}{\mathrm{d} t}+L\left(I_{1}-\frac{P_{0}}{E}\right) \frac{\mathrm{d} I_{1}}{\mathrm{~d} t}
$$

On reporte dans cette relation les expressions des dérivées de $V_{\mathrm{c}}$ et $I_{1}$ données par le système d'équations (19).

$$
\frac{\mathrm{d} V}{\mathrm{~d} t}=\left(V_{\mathrm{c}}-E\right)^{2}\left(-\frac{1}{R}+\frac{P_{0}}{E V_{\mathrm{c}}}\right)
$$




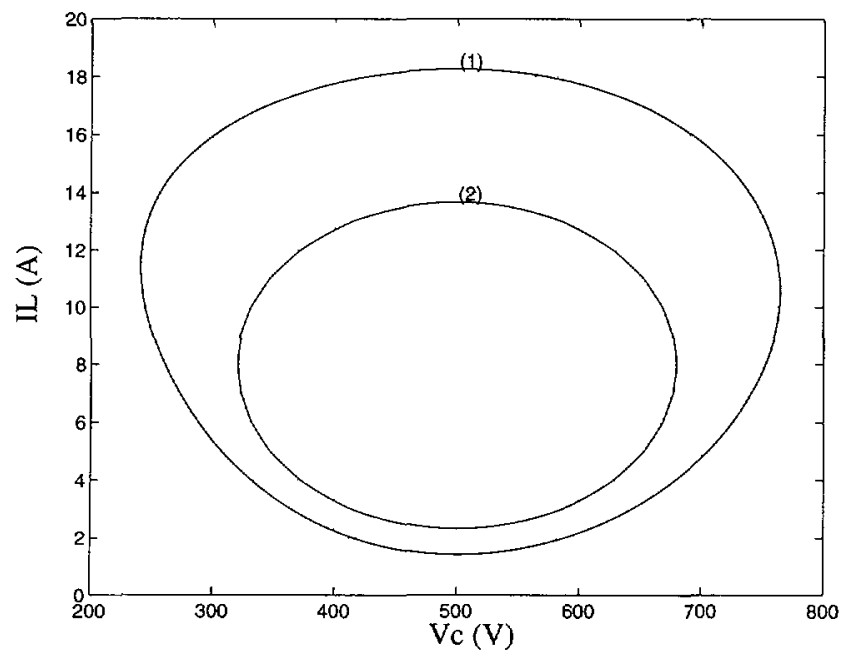

Fig. 10. - Cycle limite théorique.

[ Theoretical limit cycle.]

Il s'agit alors de déterminer le contour $V=K$ le plus grand pour lequel $V$ étant forcément positive, $\frac{\mathrm{d} V}{\mathrm{~d} t}$ soit négative. D'après l'équation (22), cela est vérifié pour :

$$
V_{c}>\frac{R P_{0}}{E}
$$

Le contour $V=K$ le plus grand est alors donné pour :

$$
\left\{\begin{array}{c}
V_{c}=\frac{R P_{0}}{E} \\
I_{1}=0
\end{array}\right.
$$

En reportant ces valeurs de $V_{c}$ et $I_{1}$ dans l'expression (20), on obtient :

$$
K=V=\frac{1}{2} C\left(\frac{R P_{0}}{E}-E\right)^{2}
$$

Le contour (2) (Fig. 10) correspond au contour établi par l'équation (25). On constate que le cycle établi par les fonctions de Ljapunov permet de définir analytiquement une grande partie du cycle réel, mais non l'intégralité de ce dernier. L'intérêt de cette méthode reste cependant de permettre la définition d'une aire de fonctionnement dans laquelle on est en mesure de garantir la stabilité du système. Cette aire est définie à partir de l'équation (25), grâce à laquelle on est en mesure de donner à $R$ et $C$ les valeurs qui permettront de fixer a priori un domaine stabilité, étant entendu que le cycle limite de stabilité obtenu par résolution numérique (qui est le cycle limite effectif) sera de dimension plus importante. 


\section{Conclusion}

Dans la mesure où le convertisseur statique d'un ensemble filtre plus convertisseur peut être représenté par une source de courant, dont la valeur est asservie à l'évolution de la tension de sortie du filtre, nous avons développé, pour l'étude de la stabilité, deux modèles. Par l'étude du modèle petit signal, il est possible d'établir des critères de stabilité liant entre eux les paramètres du circuit. Ces critères n'étant pas suffisants pour se prononcer sur la stabilité globale du système, nous avons également développé un modèle fort signal, permettant de tracer le cycle limite de stabilité. Nous avons alors montré, à l'aide des fonctions de Ljapunov, comment définir analy tiquement une zone de fonctionnement stable du système, afin de pouvoir effectuer un travail de dimensionnement du filtre d'entrée.

\section{Bibliographie}

[1] Sokal N.O., System oscillations caused by negative input resistance at the porver input port of a switching mode regulator, amplifier, $\mathrm{dc} / \mathrm{dc}$ converter, or $\mathrm{dc} / \mathrm{ac}$ inverter, IEEE Publication 73 CHO 787-2 AES, IEEE Power Electronics Specialists Conference Record (1978) 138-140.

[2] Middlebrook R.D., Input filter considerations in design and applications of switching mode regulators, IEEE Publication 76CH1122-1-IA, IEEE Indus. Appl. Soc. Annual Meeting (1976) 366-382.

[3] Polivka W.M et Erich S.Y., Input filter design criteria for current programmed regulators. IEEE Trans. Power Electron. 7 (1992) 143-151.

[4] Bickart T.A. et Balabanian N., "Electrical network theory", John Wiley \& Sons, Inc. (1969).

[5] Venable H., Minimizing input filter requirement. power supply design. 1987.PCIM (May 1987) p. 26-37.

[6] Naslin P., "Les régimes variables dans les systèmes linéaires et non linéaires", (Dunod, 1962).

[7] Pelegrin M., Gille J.-C. et Decaulne P., "Systèmes asservis non linéaires", (Dunod, 1960). 